\title{
Study of charmoniumlike and fully-charm tetraquark spectroscopy
}

\author{
Zheng Zhao, ${ }^{1, *}$ Kai Xu, ${ }^{1}$ Attaphon Kaewsnod $\odot,{ }^{1}$ Xuyang Liu, ${ }^{1,2}$ Ayut Limphirat๑, ${ }^{1}$ and Yupeng Yan ${ }^{1, \dagger}$ \\ ${ }^{1}$ School of Physics and Center of Excellence in High Energy Physics and Astrophysics, \\ Suranaree University of Technology, Nakhon Ratchasima 30000, Thailand \\ ${ }^{2}$ School of Physics, Liaoning University, Shenyang 110036, China
}

(Received 13 January 2021; revised 24 May 2021; accepted 8 June 2021; published 28 June 2021)

\begin{abstract}
The masses of tetraquark states of all $q c \bar{q} \bar{c}$ and $c c \bar{c} \bar{c}$ quark configurations are evaluated in a constituent quark model, where the Cornell-like potential and one-gluon exchange spin-spin coupling are employed. All model parameters are predetermined by comparing the theoretical and experimental masses of light, charmed and bottom mesons. The theoretical predictions of the charmoniumlike tetraquarks are compared with the observed $X Y Z$ states, and one tentative assignment is suggested. The work suggests that the $X(6900)$ observed by LHCb is likely to be the first radial excited fully-charm tetraquark state with $J^{P C}=1^{+-}$in the $\overline{3}_{c} \otimes 3_{c}$ configuration, and the ground and second radial excited states of fully-charm tetraquark are around 6494 and $7253 \mathrm{MeV}$ respectively.
\end{abstract}

DOI: $10.1103 /$ PhysRevD.103.116027

\section{INTRODUCTION}

The possible existence of multiquarks like tetraquarks $\left(q^{2} \bar{q}^{2}\right)$, pentaquarks $\left(q^{4} \bar{q}\right)$, dibaryon $\left(q^{6}\right)$ and baryonium $\left(q^{3} \bar{q}^{3}\right)$ and that baryons could be the combination of $q^{3}$ and $q^{4} \bar{q}$ while mesons could be made out of $q \bar{q}$ and $q^{2} \bar{q}^{2}$ were suggested at the birth of the quark model [1,2]. In the past two decades, over 20 charmoniumlike and bottomoniumlike XYZ states have been observed [3], which do not fit into the naive quark model spectrum easily. The observations of the XYZ states have inspired extensive interests of theorists in revealing their underlying structures. Listed in Table I are the masses, widths and $J^{P C}$ of these XYZ states in the $c \bar{c}$ region from the listed sources, or are taken from Particle Data Group (PDG) [4] when available. Instead of using the latest PDG naming scheme, we shall use $X$ for all neutral states, $Z_{c}$ for all charged states, and $Y$ for those $J^{P C}=1^{--}$states throughout this paper.

A number of charged charmoniumlike particles like $Z_{c}^{+}(3900), Z_{c}^{+}(4020), Z^{+}(4050), Z^{+}(4055), Z_{c}^{+}(4100)$, $Z_{c}^{+}$(4200), $Z_{c}^{+}(4250)$ and $Z_{c}^{+}$(4430) have been successively observed by experimental collaborations [3]. Obviously, those charged charmoniumlike states go beyond the conventional $c \bar{c}$-meson picture but are likely tetraquark systems $c \bar{c} u \bar{d}$ due to carrying one charge, which provides a good

\footnotetext{
zhaozheng1022@ hotmail.com

†yupeng@sut.ac.th
}

Published by the American Physical Society under the terms of the Creative Commons Attribution 4.0 International license. Further distribution of this work must maintain attribution to the author(s) and the published article's title, journal citation, and DOI. Funded by SCOAP. place for testing various phenomenological research methods of hadron physics. A systematic understanding of the internal structure of the charged charmoniumlike $Z_{c}$ states would provide new insights into the dynamics of multiquark systems as well as information for the future experimental search for the missing higher excitations in the $Z_{c}^{+}$family.

In the past decades, a number of models and improved pictures have been proposed to study the tetraquark states. We briefly discuss some representatives here and later in Sec. V.

The $\mathrm{X}(3872)$ was interpreted as a diquark-antidiquark charmoniumlike tetraquark state, and a spectrum was derived by taking the $\mathrm{X}(3872)$ as input [20]. The model was named "Type-I" diquark-antidiquark model. In this model, the diquark constituent quark masses were fixed by inputting the $\mathrm{X}(3872)$ state, and other parameters were fixed by the conventional meson spectrum and conventional baryon mass spectrum, where each diquark or quark-antiquark pair has its own parameters. Later, the $Z_{c}(3900)$ state was interpreted as a diquark-antidiquark charmoniumlike tetraquark state and its decay modes were investigated [21]. The Type-I diquarkantidiquark model was further developed, and a "Type-II" diquark-antidiquark model was proposed, which has more complicated spin-spin interactions [22].

The diquark-antidiquark picture was applied to study the $Z_{c}(3900) / Z_{c}(3885)$ and $Z_{c}(4020) / Z_{c}(4025)$ states [23], where the parameters were fitted by inputting the mass of $X(3823), Z_{c}(3900)$ and $Z_{c}(3885)$ states. In Ref. [23], the $Z_{c}(3900)$ and $Z_{c}(4025)$ were assigned as $Q \bar{q}-\bar{Q} q$ molecularlike four quark states, and the $Z_{c}(3885)$ as a diquarkantidiquark tetraquark state.

Within the framework of the color-magnetic interaction, the mass spectra of the hidden-charm and hidden-bottom 
TABLE I. Masses, widths and $J^{P C}$ of $\mathrm{X}, \mathrm{Y}$ and $\mathrm{Z}$ states in the $c \bar{c}$ region from the cited sources or [4] when available.

\begin{tabular}{|c|c|c|c|c|c|c|}
\hline States & Name in PDG & $\mathrm{M}(\mathrm{MeV})$ & $\Gamma$ & $J^{P C}$ & Process & Experiment \\
\hline$X(3860)$ & $\chi_{c 0}(3860)$ & $3862_{-32-13}^{+26+40}$ & $201_{-67-82}^{+154+88}$ & $0^{++}$ & $e^{+} e^{-} \rightarrow J / \psi(D \bar{D})$ & Belle [5] \\
\hline$X(3915)$ & $X(3915)$ & $3918.4 \pm 1.9$ & $\begin{array}{l}20 \pm 5 \\
20 \pm-82\end{array}$ & $0 / 2^{++}$ & $B \rightarrow K(J / \psi \omega)$ & Belle [6] \\
\hline$X(3940)$ & $X(3940)$ & $3942_{-6}^{+7} \pm 6$ & $37_{-15}^{+26} \pm 18$ & $? ?$ & $e^{+} e^{-} \rightarrow J / \psi\left(D \bar{D}^{*}\right)$ & Belle [7] \\
\hline$X(4160)$ & $X(4160)$ & $4156_{-20}^{+25} \pm 15$ & $139_{-61}^{+111} \pm 21$ & $? ?$ & $e^{+} e^{-} \rightarrow J / \psi\left(D^{*} \bar{D}^{*}\right)$ & Belle [7] \\
\hline$X(4350)$ & $X(4350)$ & $4350.6_{-51}^{+4.6} \pm 0.7$ & $13_{-9}^{+18} \pm 4$ & $?++$ & $\gamma \gamma \rightarrow \phi J / \psi$ & Belle [8] \\
\hline$X(4500)$ & $\chi_{c 0}(4500)$ & $4506 \pm 11_{-15}^{+12}$ & $92 \pm 21_{-20}^{+21}$ & $0^{++}$ & $B^{+} \rightarrow(J / \psi \phi) K^{+}$ & $\mathrm{LHCb}[9]$ \\
\hline$X(4700)$ & $\chi_{c 0}(4700)$ & $4704 \pm 10_{-24}^{+14}$ & $120 \pm 31_{-33}^{+42}$ & $0^{++}$ & $B^{+} \rightarrow(J / \psi \phi) K^{+}$ & LHCb [9] \\
\hline$Y(4230)$ & $\psi(4230)$ & $4218_{-4}^{+5}$ & $59_{-10}^{+12}$ & $1^{--}$ & $e^{+} e^{-} \rightarrow \omega \chi_{c 0}$ & BESIII [10] \\
\hline$Y(4260)$ & $\psi(4260)$ & $4230 \pm 8$ & $55 \pm 19$ & $1^{--}$ & $e^{+} e^{-} \rightarrow \pi^{+} \pi^{-} J / \psi$ & $B A B A R[11$ \\
\hline$Y(4360)$ & $\psi(4360)$ & $4368 \pm 13$ & $96 \pm 7$ & $1^{--}$ & $e^{+} e^{-} \rightarrow \pi^{+} \pi^{-} \psi(2 S)$ & BESIII [12] \\
\hline$Y(4390)$ & $\psi(4390)$ & $4391.5_{-68}^{+6.3} \pm 1.0$ & $139.5_{-206}^{+16.2} \pm 0.6$ & $1^{--}$ & $e^{+} e^{-} \rightarrow h_{c} \pi^{+} \pi^{-}$ & BESIII [13] \\
\hline$Y(4660)$ & $\psi(4660)$ & $4643 \pm 9$ & $72 \pm 11$ & $1^{--}$ & $e^{+} e^{-} \rightarrow \pi^{+} \pi^{-} \psi(2 S)$ & Belle [14] \\
\hline$Z_{c}(3900)$ & $Z_{c}(3900)$ & $3888.4 \pm 2.5$ & $28.3 \pm 2.5$ & $1^{+-}$ & $e^{+} e^{-} \rightarrow\left(D \bar{D}^{*}\right)^{+} \pi^{-}$ & BESIII [15] \\
\hline$Z_{c}(4020)$ & $X(4020)^{ \pm}$ & $4024.1 \pm 1.9$ & $13 \pm 5$ & $?^{?-}$ & $e^{+} e^{-} \rightarrow \pi^{-}\left(\pi^{+} h_{c}\right)$ & BESIII [16] \\
\hline$Z_{c}(4050)$ & $X(4050)^{ \pm}$ & $4051_{-40}^{+24}$ & $82_{-28}^{+50}$ & $?++$ & $\bar{B}^{0} \rightarrow K^{-}\left(\pi^{+} \chi_{c 1}\right)$ & Belle [17] \\
\hline$Z_{c}(4055)$ & $X(4055)^{ \pm}$ & $4054 \pm 3.2$ & $45 \pm 13$ & $?^{?-}$ & $e^{+} e^{-} \rightarrow \pi^{-}\left(\pi^{+} \psi(2 S)\right)$ & Belle [14] \\
\hline$Z_{c}(4100)$ & $X(4100)^{ \pm}$ & $4096 \pm 28$ & $152_{-70}^{+80}$ & $0^{++} / 1^{-+}$ & $B^{0} \rightarrow K^{+}\left(\pi^{-} \eta_{c}\right)$ & $\mathrm{LHCb}$ [18] \\
\hline$Z_{c}(4200)$ & $Z_{c}(4200)$ & $4196_{-32}^{+35}$ & $370_{-150}^{+100}$ & $1^{+-}$ & $\bar{B}^{0} \rightarrow K^{-}\left(\pi^{+} J / \psi\right)$ & Belle [19] \\
\hline$Z_{c}(4250)$ & $X(4250)^{ \pm}$ & $4248_{-50}^{+192}$ & $177_{-70}^{+320}$ & $?^{?+}$ & $\bar{B}^{0} \rightarrow K^{-}\left(\pi^{+} \chi_{c 1}\right)$ & Belle [17] \\
\hline$Z_{c}(4430)$ & $Z_{c}(4430)$ & $4478_{-18}^{+15}$ & $181 \pm 31$ & $1^{+-}$ & $\bar{B}^{0} \rightarrow K^{-}\left(\pi^{+} J / \psi\right)$ & Belle [19] \\
\hline
\end{tabular}

tetraquark states were studied together [24], where the constituent quark mass was fixed by charmonium meson spectrum, but the other parameters were fixed by inputting the masses of newly observed $Z_{c}(3900), Z_{c}(4025)$ and $Z_{c}(4200)$ states.

In this work we will not pay much attention to repeating the mass of potential tetraquark states by applying a large number of parameters but apply a simple model, where all the model parameters are predetermined by the conventional mesons, to predict the mass of all possible tetraquark configurations. The paper is organized as follows. In Sec. II, we work out all the possible configurations of color, flavor, spin, and spatial degrees of freedom of tetraquark states. The constituent quark model applied in our previous work [25] is briefly reviewed in Sec. III, and all model parameters are determined by comparing the theoretical and experimental masses of light, charmed and bottom mesons. In Sec. IV, tetraquark mass spectra are evaluated in the constituent quark model with all parameters predetermined. In Sec. V, the theoretical results are compared with experimental data and one tentative assignment for charmoniumlike tetraquarks is suggested. A summary is given in Sec. VI. The details of the construction of tetraquark wave functions are shown in the Appendix.

\section{CONSTRUCTION OF WAVE FUNCTION}

\section{A. Quark configurations of tetraquark}

The construction of tetraquark states follows the rule that a tetraquark state must be a color singlet, that is, the tetraquark color wave function must be a $[222]_{1}$ singlet of the $S U_{c}(3)$ group. The permutation symmetry of the two quarks cluster $(q q)$ of tetraquark states is characterized by the Young tabloids $[2]_{6},[11]_{\overline{3}}$ of the $S U_{c}(3)$ group while the color part of the two antiquarks cluster $(\bar{q} \bar{q})$ is a $[211]_{3}$ triplet and $[22]_{\overline{6}}$ antisextet, thus a $[222]_{1}$ color singlet of tetraquark states demands the following configurations:

$$
\begin{gathered}
{[2]_{6}\left(q_{1} q_{2}\right) \otimes[22]_{\overline{6}}\left(\bar{q}_{3} \bar{q}_{4}\right),} \\
{[11]_{\overline{3}}\left(q_{1} q_{2}\right) \otimes[211]_{3}\left(\bar{q}_{3} \bar{q}_{4}\right),}
\end{gathered}
$$

with the corresponding dimensions being $\overline{3}_{c} \otimes 3_{c}$ and $6_{c} \otimes \overline{6}_{c}$.

For tetraquark states of four light quarks or four charm quarks, a $q q \bar{q} \bar{q}$ state must be a color singlet and antisymmetric under any permutation between identical quarks, which implies that the spatial-spin-flavor part of color part $[2]_{6}$ and $[11]_{\overline{3}}$ should be [11] and [2] states by conjugation, respectively. Listed in Table II are all the possible configurations of spatial-spin-flavor part of the $q q$ cluster.

In this work we consider only charmoniumlike $(q c \bar{q} \bar{c})$ and fully-charm tetraquark $(c c \bar{c} \bar{c})$ states. The color wave function of each tetraquark color configuration may be written in the general form

$$
\begin{gathered}
\psi_{[2]_{6}^{c}[22]_{\overline{6}}^{c}}^{q c \bar{c} \bar{c}}=\frac{1}{\sqrt{6}} \sum_{i=1}^{6} \psi_{[2]_{6}^{c} i}^{q c} \psi_{[22]_{\bar{\sigma}}^{c} i}^{\bar{q} \bar{c}}, \\
\psi_{[11]_{\overline{3}}^{q}[211]_{3}^{c}}^{q c \bar{c}}=\frac{1}{\sqrt{3}} \sum_{i=1}^{3} \psi_{[11]_{\overline{3}}^{c} i}^{q c} \psi_{[211]_{3}^{c} i}^{\bar{q} \bar{c}}
\end{gathered}
$$


TABLE II. All possible color-spatial-spin-flavor configurations of $q q$.

\begin{tabular}{lcc}
\hline \hline$\psi_{[2]}^{c} \psi_{[2]}^{o} \psi_{[11]}^{s} \psi_{[2]}^{f}$ & $\psi_{[2]}^{c} \psi_{[11]}^{o s f}$ & \\
$\psi_{[2]}^{c} \psi_{[11]}^{o} \psi_{[11]}^{s} \psi_{[11]}^{f}$ & $\psi_{[2]}^{c} \psi_{[2]}^{o} \psi_{[2]}^{s} \psi_{[11]}^{f}$ \\
& $\psi_{[11]}^{c} \psi_{[2]}^{o s f}$ & \\
$\psi_{[11]}^{c} \psi_{[11]}^{o} \psi_{[2]}^{s} \psi_{[11]}^{f} \psi_{[2]}^{s} \psi_{[2]}^{f}$ \\
$\psi_{[11]}^{c} \psi_{[2]}^{o} \psi_{[11]}^{s} \psi_{[11]}^{f}$ & $\psi_{[11]}^{c} \psi_{[11]}^{o} \psi_{[11]}^{s} \psi_{[2]}^{f}$ \\
\hline \hline
\end{tabular}

which are for the color sextet-antisextet $\left(6_{c} \otimes \overline{6}_{c}\right)$ and triplet-antitriplet $\left(\overline{3}_{c} \otimes 3_{c}\right)$ configurations, respectively. The explicit color wave functions are listed in the Appendix. The possible spin combinations are $\left[\psi_{[s=1]}^{q c} \otimes\right.$ $\left.\psi_{[s=1]}^{\bar{q} \bar{c}}\right]_{S=0,1,2}, \quad \psi_{[s=1]}^{q c} \otimes \psi_{[s=0]}^{\bar{q} \bar{c}}, \quad \psi_{[s=0]}^{q c} \otimes \psi_{[s=1]}^{\bar{q} \bar{c}}, \quad$ and $\psi_{[s=0]}^{q c} \otimes \psi_{[s=0]}^{\bar{q} \bar{c}}:$

$$
\begin{aligned}
& {\left[\psi_{[s=1]}^{q c} \otimes \psi_{[s=1]}^{\bar{q} \bar{c}}\right]_{S=0,1,2},} \\
& \psi_{[s=1]}^{q c} \otimes \psi_{[s=0]}^{\bar{q} \bar{c}}, \quad \psi_{[s=0]}^{q c} \otimes \psi_{[s=1]}^{\bar{q} \bar{c}}, \\
& \psi_{[s=0]}^{q c} \otimes \psi_{[s=0]}^{\bar{q} \bar{c}} .
\end{aligned}
$$

For $c c \bar{c} \bar{c}$ tetraquarks, the spin wave functions for $c c$ and $\bar{c} \bar{c}$ must be symmetric and antisymmetric are for $[11]_{\overline{3}}$ and $[2]_{6}$ color configurations, respectively. The explicit spin wave functions for $c c \bar{c} \bar{c}$ are listed in the Appendix.

\section{B. Spatial wave function}

We construct the complete bases by using the harmonic oscillator wave function. The corresponding symmetries of the spatial wave functions are enforced to guarantee the antisymmetric property of identical particles.

The relative Jacobi coordinates and the corresponding momenta are defined as

$$
\begin{aligned}
& \vec{x}_{1}=\frac{1}{\sqrt{2}}\left(\vec{r}_{1}-\vec{r}_{3}\right), \\
& \vec{x}_{2}=\frac{1}{\sqrt{2}}\left(\vec{r}_{2}-\vec{r}_{4}\right), \\
& \vec{x}_{3}=\frac{m_{1} \vec{r}_{1}+m_{3} \vec{r}_{3}}{m_{1}+m_{3}}-\frac{m_{2} \vec{r}_{2}-m_{4} \vec{r}_{4}}{m_{2}+m_{4}}, \\
& \vec{x}_{0}=\frac{m_{1} \vec{r}_{1}+m_{2} \vec{r}_{2}+m_{3} \vec{r}_{3}+m_{4} \vec{r}_{4}}{m_{1}+m_{2}+m_{3}+m_{4}}, \\
& \vec{p}_{i}=u_{i} \frac{d \vec{x}_{i}}{d t},
\end{aligned}
$$

where $u_{i}$ are the reduced quark masses defined as

$$
\begin{aligned}
& u_{1}=\frac{2 m_{1} m_{3}}{m_{1}+m_{3}}, \\
& u_{2}=\frac{2 m_{2} m_{4}}{m_{2}+m_{4}}, \\
& u_{3}=\frac{\left(m_{1}+m_{3}\right)\left(m_{2}+m_{4}\right)}{m_{1}+m_{2}+m_{3}+m_{4}},
\end{aligned}
$$

where $\vec{r}_{j}$ and $m_{j}$ are the coordinate and mass of the jth quark. We assign $x_{1}, x_{2}, x_{3}$ to be $\sigma_{1}, \sigma_{2}$ and $\lambda$ Jacobi coordinates, respectively.

The total spatial wave function of tetraquark, coupling among the $\sigma_{1}, \sigma_{2}$ and $\lambda$ harmonic oscillator wave functions, may take the general form,

$$
\begin{aligned}
\psi_{N L M}= & \sum_{\left\{n_{i}, l_{i}\right\}} A\left(n_{\sigma_{1}}, n_{\sigma_{2}}, n_{\lambda}, l_{\sigma_{1}}, l_{\sigma_{2}}, l_{\lambda}\right) \\
& \times \psi_{n_{\sigma_{1}} l_{\sigma_{1}}}\left(\vec{\sigma}_{1}\right) \otimes \psi_{n_{\sigma_{2}} l_{\sigma_{2}}}\left(\vec{\sigma}_{2}\right) \otimes \psi_{n_{\lambda} l_{\lambda}}(\vec{\lambda}),
\end{aligned}
$$

where $\psi_{n_{i} l_{i} m_{i}}$ are just harmonic oscillator wave functions and the sum $\left\{n_{i}, l_{i}\right\}$ is over $n_{\sigma_{1}}, n_{\sigma_{2}}$, $n_{\lambda}, l_{\sigma_{1}}, l_{\sigma_{2}}, l_{\lambda} . N, L$, and $M$ are respectively the total principle quantum number, total angular momentum, and magnetic quantum number of tetraquark. One has $N=\left(2 n_{\sigma_{1}}+l_{\sigma_{1}}\right)+\left(2 n_{\sigma_{2}}+l_{\sigma_{2}}\right)+\left(2 n_{\lambda}+l_{\lambda}\right)$.

We will employ the spatial wave functions $\psi_{N L M}$, grouped according to the permutation symmetry, as complete bases to study tetraquark states with other interactions. In our calculations, the bases size is $N=18$, and the length parameter of harmonic oscillator wave functions is adjusted to $450 \mathrm{MeV}$ to get the best eigenvalue of Eq. (8). The complete bases of the tetraquarks are listed in the Appendix, Table XII, up to $N=10$, where $l_{\sigma_{1}}, l_{\sigma_{2}}$, and $l_{\lambda}$ are limited to 0 only.

\section{THEORETICAL MODEL}

We study the meson and tetraquark systems in the nonrelativistic Hamiltonian,

$$
\begin{aligned}
H= & H_{0}+H_{\text {hyp }}^{\text {OGE }} \\
H_{0}= & \sum_{k=1}^{N}\left(\frac{1}{2} M_{k}^{\text {ave }}+\frac{p_{k}^{2}}{2 m_{k}}\right) \\
& +\sum_{i<j}^{N}\left(-\frac{3}{16} \lambda_{i}^{C} \cdot \lambda_{j}^{C}\right)\left(A_{i j} r_{i j}-\frac{B_{i j}}{r_{i j}}\right), \\
H_{\text {hyp }}= & \sum_{i<j} C_{i j} \lambda_{i}^{C} \cdot \lambda_{j}^{C} \vec{\sigma}_{i} \cdot \vec{\sigma}_{j},
\end{aligned}
$$

by solving the Schrödinger equation

$$
H|\psi\rangle=E|\psi\rangle,
$$


TABLE III. Spin-averaged masses $M_{k}^{\text {ave }}$ for various kinds of mesons. $M_{P S}$ and $M_{V}$ taken from PDG [4].

\begin{tabular}{ccccccccc}
\hline \hline Meson & $c \bar{c}$ & $b \bar{b}$ & $B_{s}$ & $B$ & $D_{s}$ & $D$ & $s \bar{s}$ & $q \bar{q}$ \\
{$[\mathrm{MeV}]$} & 3068 & 9444 & 5404 & 5314 & 2076 & 1972 & 952 & 675 \\
\hline \hline
\end{tabular}

where the tetraquark wave function $|\psi\rangle$ is expanded in the complete bases defined in Eq. (8). Here $m_{k}$ are the constituent quark masses. $M_{k}^{\text {ave }}$ denotes the spin-averaged mass as $\frac{1}{4} M_{P S}+\frac{3}{4} M_{V}$ (except for $s \bar{s}$ and $q \bar{q}$ ), with $M_{P S}$ and $M_{V}$ being the mass of ground state pseudoscalar and vector mesons from experimental data. The spin-averaged masses $M_{k}^{\text {ave }}$ for each kind of mesons are listed in Table III. The spin-averaged masses $M_{k}^{\text {ave }}$ for $s \bar{s}$ and $q \bar{q}$ are fitted by comparing theoretical results and experimental data to avoid the would-be Goldstone bosons of the chiral symmetry breaking [3].

The Cornell potential

$$
V(r)=A r-\frac{B}{r}
$$

has been widely employed in quark model studies and also in lattice QCD simulations. Usually, the coefficient of string tension $A$ and Coulomb coefficient $B$ are fitted to experimental data. In quark model studies, model parameters are different for different hadrons, which also happens in lattice QCD studies [26,27].

The average velocity of large quark mass is predicted by the Cornell potential to saturate at the value $\left\langle v^{2}\right\rangle=B^{2}$, where B is the Coulomb coefficient in Cornell potential. According to the approximate equality of bottomonia and charmonia level splittings, $\left\langle v^{2}\right\rangle_{\Upsilon} /\left\langle v^{2}\right\rangle_{J / \psi} \approx m_{c} / m_{b}$ would be expected [28]. Thus, the Coulomb coefficients are mass dependent for large quark mass take the form $B_{b \bar{b}}=B_{c \bar{c}} \sqrt{m_{c} / m_{b}}$.

In Ref [27], interquark potentials $\left(V_{q \bar{q}}\right)$ at finite quark mass have been studied for heavy quarkonia in lattice QCD. The Cornell potential is employed for fitting the data. The fit results of Coulomb coefficient are mass dependent with the finite quark mass $\left(m_{i}\right)$ from 1.0 to $3.6 \mathrm{GeV}$, which take the form $B=B_{0} \sqrt{1 / m_{i}}$.

Later, another interquark potential study for $q \bar{q}$ systems has been done in a quenched lattice QCD [26]. One of the fitting functions is of Cornell-type, $V(r)=$ $A r-B / r+C$. The summarized fitting results show that Coulomb coefficients are mass dependent with constant quark masses $m_{q}$ which are from 0.52 to $1.275 \mathrm{GeV}$ and determined by half of vector meson masses $M_{V}$, i.e., $m_{q}=M_{V} / 2$. The mass-dependent Coulomb coefficients take the form $B=B_{0} \sqrt{1 / m_{q}}$. Meanwhile, the coefficient of string tension are linear mass dependent with the form, $A=a+b m_{q}$.
In line with the works discussed above, we propose $A_{i j}$ and $B_{i j}$ are mass-dependent coupling parameters in this work, taking the form

$$
A_{i j}=a+b m_{i j}, \quad B_{i j}=B_{0} \sqrt{\frac{1}{m_{i j}}},
$$

with $m_{i j}$ being the reduced mass of $i$ th and $j$ th quarks, defined as $m_{i j}=\frac{2 m_{i} m_{j}}{m_{i}+m_{j}} . a, b$, and $B_{0}$ are constants.

We propose the hyperfine interaction, $H_{\text {hyp }}$, has the same mass dependence as the Coulomb-like interaction in Cornell potential, assuming that the hyperfine interaction and Coulomb-like interaction are from the same route of one gluon exchange. The mass-dependent hyperfine coefficient $C_{i j}$ takes the form

$$
C_{i j}=C_{0} \sqrt{\frac{1}{m_{i j}}}
$$

where $C_{0}$ is a constant. $\lambda_{i}^{C}$ and $\vec{\sigma}_{i}$ in Eq. (7) are the quark color operator and the spin operator respectively. The color matrix elements for the configurations in Eq. (2) are summarized in Table IV. We calculate the mass spectra of light, strange, charmed and bottom mesons which are believed mainly $q \bar{q}$ states in the Hamiltonian in Eq. (7). The comparison of the theoretical results, as shown in Table V, with the experimental data taken from Particle Data Group [4] leads to the four constituent quark masses and four model coupling parameters as follows:

$$
\begin{aligned}
m_{u} & =m_{d}=420 \mathrm{MeV}, \quad m_{s}=550 \mathrm{MeV}, \\
m_{c} & =1270 \mathrm{MeV}, \quad m_{b}=4180 \mathrm{MeV} \\
a & =67413 \mathrm{MeV}^{2}, \quad b=35 \mathrm{MeV} \\
B_{0} & =31.6635 \mathrm{MeV}^{1 / 2}, \quad C_{0}=-188.765 \mathrm{MeV}^{3 / 2} .
\end{aligned}
$$

We will apply the Hamiltonian in Eq. (7) with the predetermined as well as imported parameters to predict the mass of tetraquark states.

TABLE IV. Color matrix elements of tetraquarks.

\begin{tabular}{lcc}
\hline \hline$\hat{O}$ & $\left\langle\psi_{6-\overline{6}}^{c}|\hat{O}| \psi_{6-\overline{6}}^{c}\right\rangle$ & $\left\langle\psi_{\overline{3}_{-3}}^{c}|\hat{O}| \psi_{\overline{3}_{-3}}^{c}\right\rangle$ \\
\hline$\vec{\lambda}_{1} \cdot \vec{\lambda}_{2}$ & $4 / 3$ & $-8 / 3$ \\
$\vec{\lambda}_{1} \cdot \vec{\lambda}_{3}$ & $-10 / 3$ & $-4 / 3$ \\
$\vec{\lambda}_{1} \cdot \vec{\lambda}_{4}$ & $-10 / 3$ & $-4 / 3$ \\
$\vec{\lambda}_{2} \cdot \vec{\lambda}_{3}$ & $-10 / 3$ & $-4 / 3$ \\
$\vec{\lambda}_{2} \cdot \vec{\lambda}_{4}$ & $-10 / 3$ & $-4 / 3$ \\
$\vec{\lambda}_{3} \cdot \vec{\lambda}_{4}$ & $4 / 3$ & $-8 / 3$ \\
$\sum \vec{\lambda}_{i} \cdot \vec{\lambda}_{j}$ & $-32 / 3$ & $-32 / 3$ \\
\hline \hline
\end{tabular}


TABLE V. Ground and first radial excited meson states applied to fit the model parameters. The last column shows the deviation between the experimental and theoretical mean values, $D=100 \cdot\left(M^{\exp }-M^{\text {cal }}\right) / M^{\text {exp }} . M^{\exp }$ taken from PDG [4].

\begin{tabular}{lrrr}
\hline \hline Meson & $M^{\text {exp }}(\mathrm{MeV})$ & $M^{\text {cal }}(\mathrm{MeV})$ & $D(\%)$ \\
\hline$\Upsilon(1 S)$ & 9460 & 9470 & -0.1 \\
$\Upsilon(2 S)$ & 10023 & 10070 & -0.5 \\
$\eta_{b}$ & 9399 & 9408 & -0.1 \\
$\eta_{b}(2 S)$ & 9999 & 10008 & -0.1 \\
$J / \psi$ & 3097 & 3094 & 0.1 \\
$\psi(2 S)$ & 3686 & 3678 & 0.2 \\
$\psi(3 S)$ & 4040 & 4053 & -0.3 \\
$\eta_{c}$ & 2984 & 2981 & 0.1 \\
$\eta_{c}(2 S)$ & 3638 & 3565 & 2.0 \\
$B_{s}^{*}$ & 5415 & 5439 & -0.4 \\
$B_{s}^{0}$ & 5367 & 5310 & 1.1 \\
$B^{*}$ & 5325 & 5367 & -0.8 \\
$B^{0}$ & 5279 & 5221 & 1.1 \\
$D_{s}^{*}$ & 2112 & 2127 & -0.7 \\
$D_{s 1}^{*}(2700)$ & 2708 & 2733 & -0.9 \\
$D_{s}$ & 1968 & 1981 & -0.7 \\
$D^{*}(2010)^{0}$ & 2010 & 2038 & -1.4 \\
$D^{0}$ & 1870 & 1878 & -0.4 \\
$\phi(1020)$ & 1020 & 1029 & -0.9 \\
$\phi(1680)$ & 1680 & 1660 & 1.2 \\
$\rho(770)$ & 770 & 779 & -1.2 \\
$\rho(1450)$ & 1450 & 1436 & 1.0 \\
\hline \hline & & & \\
\hline
\end{tabular}

\section{PREDICTION OF TETRAQUARK MASSES OF GROUND AND LOWER RADIAL EXCITED STATES}

The spin matrix elements for the spin combinations of charmoniumlike tetraquark states, as listed in Eq. (3), and the spin combinations of fully-charm tetraquark states, as expressed in Eqs. (A6) and (A7), are summarized respectively in Tables VI and VII.

TABLE VI. Spin matrix elements of $q c \bar{q} \bar{c}$ charmoniumlike tetraquark states.

\begin{tabular}{lrrrrr}
\hline \hline$\hat{O}$ & $\psi_{0 \otimes 0}^{S=0}$ & $\psi_{1 \otimes 1}^{S=0}$ & $\psi_{1 \otimes 0}^{S=1}$ & $\psi_{1 \otimes 1}^{S=1}$ & $\psi_{1 \otimes 1}^{S=2}$ \\
\hline$\vec{\sigma}_{1} \cdot \vec{\sigma}_{2}$ & -3 & 1 & 1 & 1 & 1 \\
$\vec{\sigma}_{1} \cdot \vec{\sigma}_{3}$ & 0 & -2 & 0 & -1 & 1 \\
$\vec{\sigma}_{1} \cdot \vec{\sigma}_{4}$ & 0 & -2 & 0 & -1 & 1 \\
$\vec{\sigma}_{2} \cdot \vec{\sigma}_{3}$ & 0 & -2 & 0 & -1 & 1 \\
$\vec{\sigma}_{2} \cdot \vec{\sigma}_{4}$ & 0 & -2 & 0 & -1 & 1 \\
$\vec{\sigma}_{3} \cdot \vec{\sigma}_{4}$ & -3 & 1 & -3 & 1 & 1 \\
$\sum \vec{\sigma}_{i} \cdot \vec{\sigma}_{j}$ & -6 & -6 & -2 & -2 & 6 \\
\hline \hline
\end{tabular}

TABLE VII. Spin matrix elements of $c c \bar{c} \bar{c}$ fully-charm tetraquark states.

\begin{tabular}{lcccc}
\hline \hline$\hat{O}$ & $\psi_{(6 \otimes \overline{6})(0 \otimes 0)}^{C, S=0}$ & $\psi_{(\overline{3} \otimes 3)(1 \otimes 1)}^{C, S=0}$ & $\psi_{(\overline{3} \otimes 3)(1 \otimes 1)}^{C, S=1}$ & $\psi_{(\overline{3} \otimes 3)(1 \otimes 1)}^{C, S=2}$ \\
\hline$\vec{\sigma}_{1} \cdot \vec{\sigma}_{2}$ & -3 & 1 & 1 & 1 \\
$\vec{\sigma}_{1} \cdot \vec{\sigma}_{3}$ & 0 & -2 & -1 & 1 \\
$\vec{\sigma}_{1} \cdot \vec{\sigma}_{4}$ & 0 & -2 & -1 & 1 \\
$\vec{\sigma}_{2} \cdot \vec{\sigma}_{3}$ & 0 & -2 & -1 & 1 \\
$\vec{\sigma}_{2} \cdot \vec{\sigma}_{4}$ & 0 & -2 & -1 & 1 \\
$\vec{\sigma}_{3} \cdot \vec{\sigma}_{4}$ & -3 & 1 & 1 & 1 \\
$\sum \vec{\sigma}_{i} \cdot \vec{\sigma}_{j}$ & -6 & -6 & -2 & 6 \\
\hline \hline
\end{tabular}

TABLE VIII. Ground and first radial excited charmoniumlike tetraquark masses $(\mathrm{MeV})$.

\begin{tabular}{llcc}
\hline \hline$q c \bar{q} \bar{c}$ configurations & \multicolumn{1}{c}{$I^{G} J^{P C}$} & $\mathrm{M}(1 \mathrm{~S})$ & $\mathrm{M}(2 \mathrm{~S})$ \\
\hline$\left.\Psi_{(6 \otimes \overline{6}}^{c s}\left[(q c)^{s=0} \otimes(\bar{q} \bar{c})^{s=0}\right)\right]^{S=0}$ & $0^{+} 0^{++} / 1^{-} 0^{++}$ & 4202 & 4566 \\
$\Psi_{\left.(\overline{3} \otimes 3)\left[(q c)^{s=0} \otimes(\bar{q} \bar{c})^{s=0}\right)\right]^{S=0}}^{c s}$ & $0^{+} 0^{++} / 1^{-} 0^{++}$ & 4033 & 4434 \\
$\Psi_{\left.(6 \otimes \overline{6})\left[(q c)^{s=1} \otimes(\bar{q} \bar{c})^{s=1}\right)\right]^{S=0}}^{c s}$ & $0^{+} 0^{++} / 1^{-} 0^{++}$ & 3925 & 4289 \\
$\Psi_{\left.(\overline{3} \otimes 3)\left[(q c)^{s=1} \otimes(\bar{q} \bar{c})^{s=1}\right)\right]^{S=0}}^{c s}$ & $0^{+} 0^{++} / 1^{-} 0^{++}$ & 4114 & 4516 \\
$\Psi_{\left.(6 \otimes \overline{6})\left[(q c)^{s=1} \otimes(\bar{q} \bar{c})^{s=0}\right)\right]^{S=1}}^{c s}$ & $0^{-} 1^{+-} / 1^{+} 1^{+-}$ & 4162 & 4526 \\
$\Psi_{\left.(\overline{3} \otimes 3)\left[(q c)^{s=1} \otimes(\bar{q} \bar{c})^{s=0}\right)\right]^{S=1}}$ & $0^{-} 1^{+-} / 1^{+} 1^{+-}$ & 4113 & 4514 \\
$\left.\Psi_{(6 \otimes \overline{6}}^{c s}\left[(q c)^{s=1} \otimes(\bar{q} \bar{c})^{s=1}\right)\right]^{S=1}$ & $0^{-} 1^{+-} / 1^{+} 1^{+-}$ & 4024 & 4388 \\
$\Psi_{\left.(\overline{3} \otimes 3)\left[(q c)^{s=1} \otimes(\bar{q} \bar{c})^{s=1}\right)\right]^{S=1}}^{c s}$ & $0^{-} 1^{+-} / 1^{+} 1^{+-}$ & 4154 & 4555 \\
$\Psi_{\left.\left.(6 \otimes \overline{6})\left[(q c)^{s=1} \otimes(\bar{q} \bar{c})^{s=1}\right)\right]^{S=2}\right)}^{c s}$ & $0^{+} 2^{++} / 1^{-} 2^{++}$ & 4221 & 4584 \\
$\Psi_{\left.(\overline{3} \otimes 3)\left[(q c)^{s=1} \otimes(\bar{q} \bar{c})^{s=1}\right)\right]^{S=2}}^{c s}$ & $0^{+} 2^{++} / 1^{-} 2^{++}$ & 4233 & 4634 \\
\hline \hline
\end{tabular}

The mass spectra of the ground and first radial excited charmoniumlike tetraquarks and the ground, first and second radial excited fully-charm tetraquarks are evaluated in the Hamiltonian in Eq. (7), where the complete bases defined in Sec. II are applied and the model parameters are predetermined in Sec. III. Listed in Tables VIII and IX are the theoretical results for

TABLE IX. Ground, first and second radial excited fully-charm tetraquark masses $(\mathrm{MeV})$.

\begin{tabular}{llccc}
\hline \hline$c c \bar{c} \bar{c}$ configurations & $I^{G} J^{P C}$ & $\mathrm{M}(1 \mathrm{~S})$ & $\mathrm{M}(2 \mathrm{~S})$ & $\mathrm{M}(3 \mathrm{~S})$ \\
\hline$\Psi_{\left.(6 \otimes \overline{6})\left[(c c)^{s=0} \otimes(\bar{c} \bar{c})^{s=0}\right)\right]^{S=0}}^{c s}$ & $0^{+} 0^{++}$ & 6514 & 6840 & 7098 \\
$\Psi_{\left.(\overline{3} \otimes 3)\left[(c c)^{s=0} \otimes(\bar{c} \bar{c})^{s=0}\right)\right]^{S=0}}^{c S}$ & $0^{+} 0^{++}$ & 6466 & 6883 & 7225 \\
$\Psi_{\left.(\overline{3} \otimes 3)\left[(c c)^{s=1} \otimes(\bar{c} \bar{c})^{s=1}\right)\right]^{S=1}}^{c s}$ & $0^{-} 1^{+-}$ & 6494 & 6911 & 7253 \\
$\Psi_{\left.(\overline{3} \otimes 3)\left[(c c)^{s=1} \otimes(\bar{c} \bar{c})^{s=1}\right)\right]^{S=2}}^{c s}$ & $0^{+} 2^{++}$ & 6551 & 6968 & 7310 \\
\hline \hline
\end{tabular}


charmoniumlike and fully-charm tetraquarks of various quark configurations, respectively.

\section{DISCUSSION}

As a number of exotic particles have been discovered, recent years can be called a revolutionary period in the field of hadron physics. Among the exotic particles, charmoniumlike charged mesons may be especially interesting since they have a charmoniumlike mass but are electrically charged [29]. Those charged charmoniumlike states go beyond the conventional $c \bar{c}$-meson picture and are likely tetraquark states $c \bar{c} u \bar{d}$ due to carrying one charge. We have listed these states in Table I, denoting all of them with X, Y and $Z_{c}$, which are more convenient for referring to other works than the latest naming scheme of the Particle Data Group [4]. We shall use $X$ and $Z_{c}$, throughout this discussion, to refer to neutral states and charged states with hidden charm respectively.

\section{A. Charmoniumlike tetraquark states}

$Z_{c}(4050)$ and $Z_{c}(4250)$ have been studied as tetraquarks in different models. $Z_{c}(4050)$ was investigated as a cluster of $Q \bar{q}$ and $\bar{Q} q$ in a Cornell-like potential with some residual color forces that bind the two clusters [23], which results in two states with masses 4046 and $4054 \mathrm{MeV}$ with quantum numbers $J^{P C}=2^{+-}$and $J^{P C}=3^{++}$respectively. Both states are associated with $Z_{c}(4050)$. In the color flux-tube model, a conclusion was made that $Z_{c}(4050)$ has a tetraquark $(c u)(\bar{c} \bar{d})$ nature, with spin-parity $J^{P}=1^{-}$, while $Z_{c}(4250)$ could be interpreted as a $(c u)(\bar{c} \bar{d})$ tetraquark with $J^{P}=1^{+}[30]$. No tetraquark candidate was found for $Z_{c}(4050)$ in a relativistic quark model, but $Z_{c}(4250)$ could be interpreted as a tetraquark state [31].

Considering that both $Z_{c}(4050)$ and $Z_{c}(4250)$ were observed in the process $\bar{B}^{0} \rightarrow K^{-} \pi^{+} \chi_{c 1}$ and their tentative quantum numbers [17], the present predictions support assigning the $Z_{c}(4050)$ and $Z_{c}(4250)$ to be the ground states with $J^{P C}=0^{++}$of the $\left(\overline{3}_{c} \otimes 3_{c}\right)\left(0_{s} \otimes 0_{s}\right)_{S=0}$ and $\left(6_{c} \otimes \overline{6}_{c}\right)\left(0_{s} \otimes 0_{s}\right)_{S=0}$ configurations, respectively.

The tetraquark nature of $Z_{c}(4200)$ has been studied in various model calculations as well. A $(c u)(\bar{c} \bar{d})$ state was predicted, with the quantum numbers $n\left({ }^{2 S+1} L_{J}\right)=1\left({ }^{3} D_{1}\right)$ and spin-parity $1^{+}$, which is associated with $Z_{c}(4200)$, in a model treating quark-quark interactions through one gluon exchange, one boson exchange and $\sigma$ exchange [32]. The study in a light-front holographic QCD model preferred that $Z_{c}(4200)$ having a generic dilaton profile [33]. Using a formalism based on color magnetic interactions, $Z_{c}(4200)$ was described as an axial vector tetraquark state [24].

After its discovery, the $Z_{c}^{+}(3900)$ was identified as the predicted $X^{+}$[21], and $Z^{+}(4430)$ was identified as the first radial excitation of $Z_{c}^{+}$(3900) [22]. $Z_{c}^{+}$(4430) was also interpreted as the first radial excitation $(2 \mathrm{~S})$ of a charged diquark-antidiquark $(c u)(\bar{c} \bar{d})$ tetraquark state in Refs. [23,31,34-36].

Since both $Z_{c}(4200)$ and $Z_{c}(4430)$ were observed in the process $\bar{B}^{0} \rightarrow K^{-} \pi^{+} J / \psi$ [19] and their decay widths are in the same order which is much larger than the $Z_{c}^{+}(3900)$ one, one may naturally pair the $Z_{c}(4200)$ and $Z_{c}(4430)$ together. Therefore, we may assign the $Z_{c}(4200)$ and $Z_{c}(4430)$ to be the ground and first radial excited states, with $J^{P C}=1^{+-}$, of the $\left(6_{c} \otimes \overline{6}_{c}\right)\left(1_{s} \otimes 0_{s}\right)_{S=1}$ configuration, respectively.

In the color flux tube model with a multibody confinement potential, tetraquark states were studied in the diquark-antidiquark configuration, and it was found that the nearest state to $Z_{c}^{+}(4025)$ obtained by the model is the one with quantum number $J^{P}=2^{+}[30,37]$. However, more works support the $1^{+-}$quantum numbers. In the framework of nonrelativistic quark model and applying a Cornell-type potential, a molecularlike four-quark state of $Q \bar{q}-\bar{Q} q$ with $J^{P C}=1^{+-}$was predicted around $4036 \mathrm{MeV}$, among others with similar masses but other quantum numbers [23]. This state was identified with $Z_{c}(4025) . Z_{c}(4025)$ and $Z_{c}(4020)$ are named as $X(4020)$ nowadays in PDG [4]. Except for the parity, other quantum numbers of the $X(4020)$ are not well determined, but all the experimental analyses from BESIII assumed s-wave productions and the quantum number assignment $J^{P C}=1^{+-}[16,38-40]$.

Belle updated the measurement of $e^{+} e^{-} \rightarrow \pi^{+} \pi^{-} \psi(2 S)$ via initial state radiation using the $980 \mathrm{fb}^{-1}$ full data sample [14]. Figure 1 showed the $M_{\max }\left[\pi^{ \pm}(2 S)\right]$ distribution, the maximum of $M\left[\pi^{-} \psi(2 S)\right]$ and $M\left[\pi^{+} \psi(2 S)\right]$, in $Y(4360)$ decays $\left(4.0 \mathrm{GeV}<M_{\pi^{+} \pi^{-} \psi(2 S)}<4.5 \mathrm{GeV}\right)$, where an excess evidence at around $4.05 \mathrm{GeV}$ can be seen. The fit yields a mass of $(4054 \pm 3 \pm 1) \mathrm{MeV}$ and a width of $(45 \pm 11 \pm 6) \mathrm{MeV}$. The statistical significance of the signal is $3.5 \sigma$ with systematic uncertainties included.

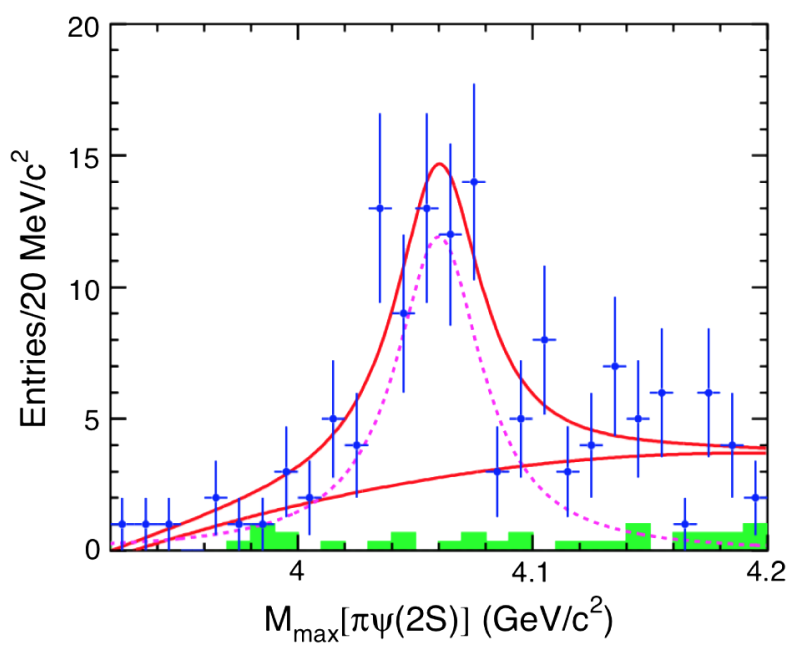

FIG. 1. The distributions of $M_{\max }\left[\pi^{ \pm} \psi(2 S)\right]$ from $Y(4360)$ decays [14]. 


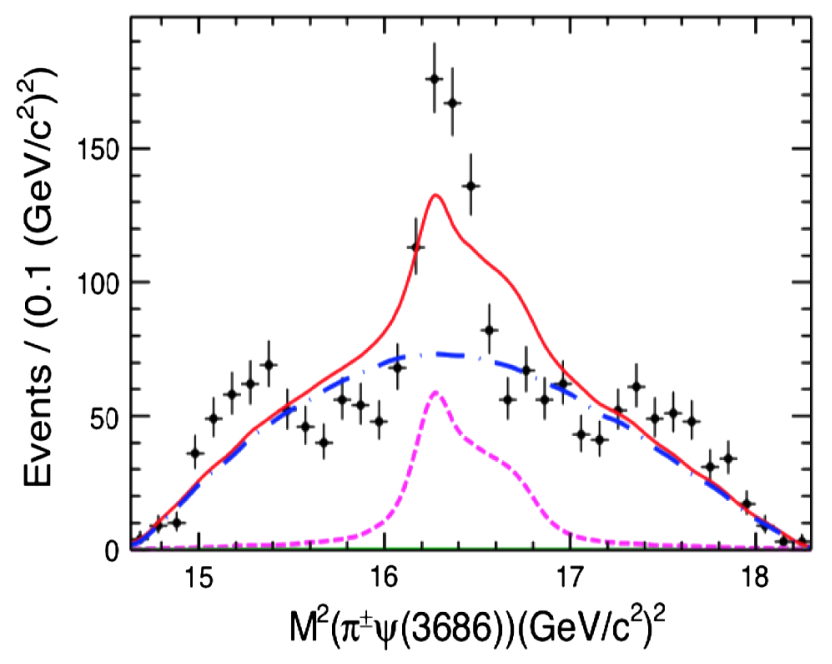

FIG. 2. The distributions of $M\left[\pi^{ \pm} \psi(2 S)\right]$ at $\sqrt{s}=4.416 \mathrm{GeV}$ (two entries per event) from BESIII [12].

BESIII studied the process $e^{+} e^{-} \rightarrow \pi^{+} \pi^{-} h_{c}$ at c.m. energies from 3.90 to $4.42 \mathrm{GeV}$ [16]. In the $\pi^{ \pm} h_{c}$ mass spectrum, a distinct structure, referred to as $Z_{c}(4020)$, was observed at $4.02 \mathrm{GeV}$. The $Z_{c}(4020)$ carries an electric charge and couples to charmonium. A fit to the $\pi^{ \pm} h_{c}$ invariant mass spectrum, neglecting possible interferences, results in a mass of $(4022.9 \pm 0.8 \pm 2.7) \mathrm{MeV}$ and a width of $(7.9 \pm 2.7 \pm 2.6) \mathrm{MeV}$ for the $Z_{c}(4020)$, where the first errors are statistical and the second systematic. Later, BESIII studied the process $e^{+} e^{-} \rightarrow \pi^{+} \pi^{-} \psi(2 S)$ using $5.1 \mathrm{fb}^{-1}$ of data at c.m. energies from 4.0 to $4.6 \mathrm{GeV}$ [12]. For data at $\sqrt{s}=4.416 \mathrm{GeV}$, a prominent narrow structure was observed around $4030 \mathrm{MeV}$ in the $M\left[\pi^{ \pm} \psi(2 S)\right]$ spectrum, as shown in Fig. 2. The fit yields a mass of $M=(4032.1 \pm 2.4) \mathrm{MeV}$ and a width of $\Gamma=(26.1 \pm 5.3) \mathrm{MeV}$ for the intermediate state with a much higher significance than Belle of $9.2 \sigma$.

The authors of Ref. [41] reported their preliminary partial wave analysis (PWA) results on $e^{+} e^{-} \rightarrow \pi^{+} \pi^{-} \psi(2 S)$ at the Charm 2018 meeting by using BESIII published results [12]. The fit quality is much improved. It was found that the structure can be described well with a charged state with a mass of $(4019.0 \pm 1.9) \mathrm{MeV}$ and a width of $(29 \pm 4) \mathrm{MeV}$, or the $Z_{c}(4020)$ state observed in the $\pi^{+} \pi^{-} h_{c}$ final state [16]. If such PWA results are confirmed in the future, one may argue that $Z_{c}(4020)$ may have another decay mode $Z_{c}(4020) \rightarrow \pi^{+} \psi(2 S)$ and furthermore $Z_{c}(4020)$ and $Z_{c}(4055)$ could be the same state. Thus, both $\pi^{+} \pi^{-} \psi(2 S)$ and $\pi^{+} \pi^{-} h_{c}$ final states need to be further investigated to understand the intermediate structures. At this moment, we may just assign either $Z_{c}(4020)$ or $Z_{c}(4055)$, if they are not the same particle, to be the $\left(6_{c} \otimes \overline{6}_{c}\right)\left(1_{s} \otimes 1_{s}\right)_{s=1}$ configuration tetraquark ground states with $J^{P C}=1^{+-}$.

Up to now, there have been only few theoretical calculations for $Z_{c}(4100)$. A simple chromomagnetic model was employed to study the mass splitting among tetraquark states, including $Z_{c}(4100)$ [42]. The model is based on the description that the mass splitting among hadron states, with the same quark content, are mainly due to the chromomagnetic interaction term in the one-gluonexchange potential. Based on these findings, it was concluded that $Z_{c}(4100)$ seems to be a $0^{++}(c q)(\bar{c} \bar{q})$ tetraquark state. In our assignments, $Z_{c}(4100)$ is assigned to be the $\left(\overline{3}_{c} \otimes 3_{c}\right)\left(1_{s} \otimes 1_{s}\right)_{S=0}$ configuration tetraquark ground state with $J^{P C}=0^{++}$.

Considering that the $X(3915)$ and $X(4350)$ have similar decay patterns, that is, the $X(3915)$ state decays mainly to $J / \psi \omega$ and the $X(4350)$ state was observed only in the process $\gamma \gamma \rightarrow \phi J / \psi$, and that their decay widths were narrow and in the same order, we may assign $X(3915)$ and $X(4350)$ together to be ground and first radial excited states, with $J^{P C}=0^{++}$, of the $\left(6_{c} \otimes \bar{\sigma}_{c}\right)\left(1_{s} \otimes 1_{s}\right)_{S=0}$ configuration, respectively.

There is no room to accommodate the $Z_{c}(3900)$ in the scenario of tetraquark states in the present model. As discussed above, there are two possible explanations for the $Z_{c}^{+}(3900)$ structure: a charged diquark-antidiquark $(c u)(\bar{c} \bar{d})$ state, and a $D \bar{D}^{*}$ molecular state. Concerning the molecular configuration, there are many calculations that could not accommodate $Z_{c}^{+}(3900)$ as a $J^{P}=1^{+} D \bar{D}^{*}$ molecule, including lattice QCD calculations [43-46]. In Refs. [47-49], however, a $D \bar{D}^{*}$ molecular state is derived, compatible with $Z_{c}^{+}(3900) . Z_{c}(3900)$ may be axial vector moleculelike state with $J^{P C}=1^{+-}$.

TABLE X. Tentative assignments of ground and first radial excited charmoniumlike tetraquark states.

\begin{tabular}{|c|c|c|c|c|c|c|c|c|c|c|}
\hline \multirow[b]{3}{*}{$q c \bar{q} \bar{c}$ configurations } & \multicolumn{2}{|c|}{$\psi_{0_{s} \otimes 0_{s}}^{S=0}$} & \multicolumn{2}{|c|}{$\psi_{1_{s} \otimes 1_{s}}^{S=0}$} & \multicolumn{2}{|c|}{$\psi_{1_{s} \otimes 0_{s}}^{S=1}$} & \multicolumn{2}{|r|}{$\psi_{1_{s} \otimes 1_{s}}^{S=1}$} & \multicolumn{2}{|c|}{$\psi_{1_{s} \otimes 1_{s}}^{S=2}$} \\
\hline & \multicolumn{2}{|c|}{$0^{+} 0^{++} / 1^{-} 0^{++}$} & \multicolumn{2}{|c|}{$0^{+} 0^{++} / 1^{-} 0^{++}$} & \multicolumn{2}{|c|}{$0^{-} 1^{+-} / 1^{+} 1^{+-}$} & \multicolumn{2}{|r|}{$0^{-} 1^{+-} / 1^{+} 1^{+-}$} & \multicolumn{2}{|c|}{$0^{+} 2^{++} / 1^{-} 2^{++}$} \\
\hline & Ours & Data & Ours & Data & Ours & Data & Ours & Data & Ours & Data \\
\hline$\Psi_{6_{c} \otimes \bar{b}_{c}}^{c}(1 S)$ & 4202 & $Z_{c}(4250)$ & 3925 & $X(3915)$ & 4162 & $Z_{c}(4200)$ & 4024 & $Z_{c}(4020) / Z_{c}(4055)$ & 4221 & $\cdots$ \\
\hline$\Psi_{\sigma_{c} \otimes \bar{b}_{c}}^{c}(2 S)$ & 4566 & $\ldots$ & 4289 & $X(4350)$ & 4526 & $Z_{c}(4430)$ & 4388 & $\ldots$ & 4584 & $\cdots$ \\
\hline$\Psi \overline{3}_{c} \otimes 3_{c}(1 S)$ & 4033 & $Z_{c}(4050)$ & 4114 & $Z_{c}(4100)$ & 4113 & $X(4160)$ & 4154 & $X(4160)$ & 4233 & $\cdots$ \\
\hline$\Psi_{\overline{\overline{3}}_{c}^{c} \otimes 3_{c}}^{c}(2 S)$ & 4434 & $\ldots$ & 4516 & $\ldots$ & 4514 & $\ldots$ & 4555 & $\cdots$ & 4634 & $\cdots$ \\
\hline
\end{tabular}




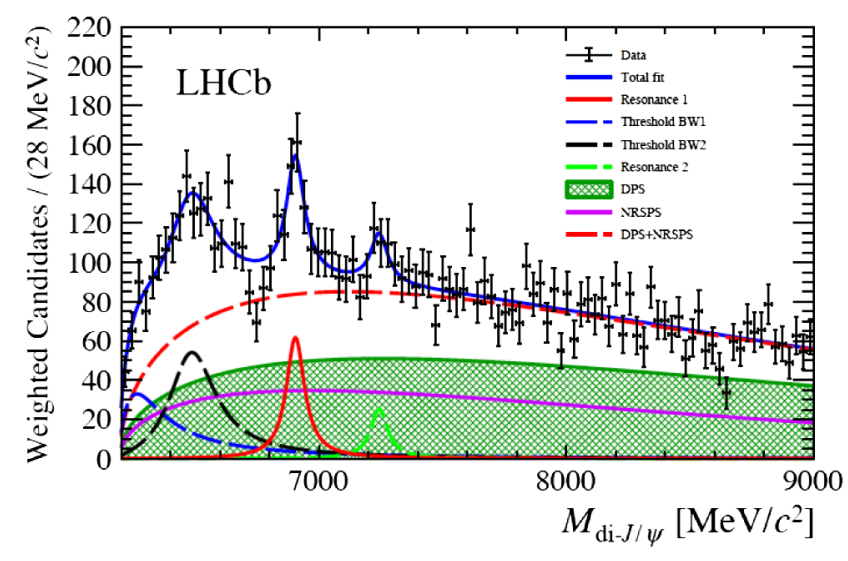

FIG. 3. Invariant mass spectra of weighted di- $J / \psi$ candidates. This was adapted from Fig. S3 in [50].

Except the assignments discussed above, $X(4160)$ is also tentatively assigned to be the $\left(\overline{3}_{c} \otimes 3_{c}\right)\left(1_{s} \otimes 0_{s}\right)_{S=1}$ and $\left(\overline{3}_{c} \otimes 3_{c}\right)\left(1_{s} \otimes 1_{s}\right)_{S=1}$ configuration tetraquark ground states with $J^{P C}=1^{+-}$according to the mass matching.

Here, in Table X, almost all the ground charmoniumlike tetraquark states predicted in the work have been tentatively matched with experimental data. Seven in eight charged charmoniumlike tetraquark states observed by experimental collaborations have been assigned in the work except $Z_{c}^{+}$(3900). More experimental data and theoretical works are essential to make unambiguous assignments. In addition, there is no room of the ground and first excited states to accommodate the $X(3860), X(3940), X(4500)$, and $X(4700)$ in the scenario of tetraquark states in the present model.

\section{B. Fully-charm tetraquark states}

The LHCb collaboration has recently presented evidence for the observation of at least one resonance in the $J / \psi$-pair spectrum at about $6900 \mathrm{MeV}$ [50]. The data also revealed a broader structure centered around $6500 \mathrm{MeV}$, as shown in Fig. 3. Such states are naturally assigned the valence-quark content $c \bar{c} c \bar{c}$, making them the first all-heavy multiquark exotic candidates claimed to date in the experimental literature. The measured mass and width of the resonance around $6900 \mathrm{MeV}$ are

$$
\begin{aligned}
M[X(6900)] & =6905 \pm 11 \pm 7 \\
\Gamma[X(6900)] & =80 \pm 19 \pm 33
\end{aligned}
$$

or, using a second fitting model

$$
\begin{aligned}
M[X(6900)] & =6886 \pm 11 \pm 11 \\
\Gamma[X(6900)] & =168 \pm 33 \pm 69 .
\end{aligned}
$$

Our present theoretical predictions, as shown in Table IX, support assigning the $X(6900)$ to be the first radial excited fully-charm tetraquark state, with $J^{P C}=1^{+-}$, in the $\overline{3}_{c} \otimes 3_{c}$ configuration. The ground and second radial excited states of fully-charm tetraquarks are around 6494 and $7253 \mathrm{MeV}$ respectively, with $J^{P C}=1^{+-}$, in the $\overline{3}_{c} \otimes 3_{c}$ configuration, which is compatible with the experimental data in Fig. 3.

We collect our numerical results and some typical results of other works in Table XI for comparison. Including our results, the predictions of nonrelativistic quark models are roughly compatible [51-55], where both confining and OGE Coulomb-like potentials are considered. One work in QCD sum rules also gives similar results [56]. References [57-61] give smaller masses, without considering color configurations.

\section{SUMMARY}

We have evaluated the masses of ground and first radial excited charmoniumlike tetraquark states and of ground and first and second radial excited states of the fully-charm tetraquark states, with all model parameters predetermined by comparing the theoretical and experimental masses of light, charmed and bottom mesons.

We have made a tentative matching between the predicted ground and first radial excited charmoniumlike tetraquark states and the $\mathrm{X}$ and $\mathrm{Z}$ particles. The present work predicts some charmoniumlike tetraquark states which cannot be matched with observed particles. One may suggest experimental searchings in the processes $e^{+} e^{-} \rightarrow \pi^{\mp}\left(\pi^{ \pm} h_{c}\right), \pi^{\mp}\left(\pi^{ \pm} \psi(2 S)\right)$ for higher mass reso-

\begin{tabular}{|c|c|c|c|c|c|c|c|c|c|c|c|c|}
\hline$c c \bar{c} \bar{c}$ configurations & $J^{P C}$ & Ours & {$[51]$} & {$[52]$} & {$[53]$} & {$[54]$} & {$[55]$} & {$[56]$} & [57] & {$[58]$} & {$[59,60]$} & [61] \\
\hline \multirow{2}{*}{$\begin{array}{l}\Psi_{\left(6_{c} \otimes \overline{6}_{c}\right)\left(0_{s} \otimes 0_{s}\right)^{S=0}} \\
\Psi_{\left(\overline{3}_{c} \otimes 3_{c}\right)\left(1_{s} \otimes 1_{s}\right)^{S=0}}^{c s}\end{array}$} & $0^{++}$ & 6514 & 6404 & $6383-6421$ & 6518 & 6695 & 6383 & $6440-6820$ & \multirow[t]{2}{*}{$6038-6115$} & \multirow[t]{2}{*}{5966} & \multirow[t]{2}{*}{5990} & \multirow[t]{2}{*}{5969} \\
\hline & $0^{++}$ & 6466 & 6421 & $6420-6436$ & 6487 & 6477 & 6437 & $6460-6470$ & & & & \\
\hline$\Psi_{\left(\overline{3}_{c} \otimes 3_{c}\right)\left(1_{s} \otimes 1_{s}\right)^{S=1}}^{c s}$ & $1^{+-}$ & 6494 & 6439 & $6425-6450$ & 6500 & 6528 & 6437 & $6370-6510$ & $6101-6176$ & 6051 & 6050 & 6021 \\
\hline$\Psi_{\left(\overline{3}_{c} \otimes 3_{c}\right)\left(1_{s} \otimes 1_{s}\right)^{S=2}}^{c s}$ & $2^{++}$ & 6551 & 6472 & $6432-6479$ & 6524 & 6573 & 6437 & $6370-6510$ & $6172-6216$ & 6223 & 6090 & 6115 \\
\hline
\end{tabular}
nances, probably the first radial excited states of $Z_{c}(4020)$, and $Z_{c}(4055)$.

TABLE XI. Present predictions of ground state fully-charm tetraquark masses $(\mathrm{MeV})$ compared with others. 
The work suggests that the $X(6900)$ observed by $\mathrm{LHCb}$ is likely the first radial excited fully-charm tetraquark state, with $J^{P C}=1^{+-}$, in the $\overline{3}_{c} \otimes 3_{c}$ configuration. The ground and second radial excited states of fully-charm tetraquarks, with $J^{P C}=1^{+-}$, in the $\overline{3}_{c} \otimes 3_{c}$ configuration are around 6494 and $7253 \mathrm{MeV}$, respectively.

\section{ACKNOWLEDGMENTS}

This work is supported by Suranaree University of Technology (SUT). Z. Zhao acknowledges support from SUT-OROG Ph.D. scholarship under Contract No. 62/ 2559. X. Y. Liu acknowledges support from the Young Science Foundation from the Education Department of Liaoning Province, China (Project No. LQ2019009).

\section{APPENDIX: CONSTRUCTION OF TETRAQUARK WAVE FUNCTIONS}

The construction of tetraquark states follows the rule that a tetraquark state must be a color singlet and the $q q$ and $\bar{q} \bar{q}$ clusters wave function should be antisymmetric under any permutation between identical quarks. Requiring the tetraquark to be a color singlet demands that the color part of the $q q$ and $\bar{q} \bar{q}$ must form a $S U_{c}(3)[222]_{1}$ singlet state, and hence two sets of color configurations, $[2]\left(q_{1} c_{2}\right) \otimes$ $[22]\left(\bar{q}_{3} \bar{c}_{4}\right)$ and $[11]\left(q_{1} c_{2}\right) \otimes[211]\left(\bar{q}_{3} \bar{c}_{4}\right)$, taking charmoniumlike tetraquark states as an example. We may take charmoniumlike tetraquark states as an example. The order of quarks is defined as $q_{1} c_{2} \bar{q}_{3} \bar{c}_{4}$ :

$[2]\left(q_{1} c_{2}\right) \otimes[22]\left(\bar{q}_{3} \bar{c}_{4}\right)$ and $\quad[11]\left(q_{1} c_{2}\right) \otimes[211]\left(\bar{q}_{3} \bar{c}_{4}\right)$,

where denote color configurations sextet-antisextet $\left(6_{c} \otimes \overline{6}_{c}\right)$ and triplet-antitriplet $\left(\overline{3}_{c} \otimes 3_{c}\right)$.

The color wave function of each tetraquark color configuration may be written in the general form,

$$
\begin{aligned}
& \psi_{[2]]_{6}^{c}[22]_{\overline{6}}^{c_{0}}}^{q_{1} c_{2} \bar{q}_{3} \bar{c}_{4}}=\frac{1}{\sqrt{6}} \sum_{i=1}^{6} \psi_{[2]_{6}^{c} c_{i} i}^{q_{1} c_{2}} \psi_{[22]_{\overline{6}} \bar{c}_{i} i}^{\bar{q}_{3} \bar{c}_{4}}, \\
& \psi_{[11]]_{3}^{c}[211]_{3}^{c}}^{q_{1} c_{2} \bar{q}_{3} \bar{c}_{4}}=\frac{1}{\sqrt{3}} \sum_{i=1}^{3} \psi_{[11]_{3}^{c}}^{q_{1} c_{2}} \psi_{[211]_{3}^{c} i}^{\bar{q}_{3} \bar{c}_{4}} .
\end{aligned}
$$

The explicit color wave functions without subscripts are using the quark order $q_{1} c_{2} \bar{q}_{3} \bar{c}_{4}$, listed as follows:

$$
\begin{aligned}
& \psi_{[2]_{6}[22]_{6}^{c}}^{q_{1} c_{2} \bar{q}_{3} \bar{c}_{4}}=\frac{1}{\sqrt{6}}\left[R_{1} R_{2} \bar{R}_{3} \bar{R}_{4}+G_{1} G_{2} \bar{G}_{3} \bar{G}_{4}+B_{1} B_{2} \bar{B}_{3} \bar{B}_{4}\right. \\
& +\frac{1}{2}\left(R_{1} G_{2}+G_{1} R_{2}\right)\left(\bar{R}_{3} \bar{G}_{4}+\bar{G}_{3} \bar{R}_{4}\right) \\
& +\frac{1}{2}\left(B_{1} R_{2}+R_{1} B_{2}\right)\left(\bar{B}_{3} \bar{R}_{4}+\bar{R}_{3} \bar{B}_{4}\right) \\
& \left.+\frac{1}{2}\left(G_{1} B_{2}+B_{1} G_{2}\right)\left(\bar{G}_{3} \bar{B}_{4}+\bar{B}_{3} \bar{G}_{4}\right)\right] \\
& =\frac{1}{\sqrt{6}}[R R \bar{R} \bar{R}+G G \bar{G} \bar{G}+B B \bar{B} \bar{B} \\
& +\frac{1}{2}(R G \bar{R} \bar{G}+G R \bar{R} \bar{G}+R G \bar{G} \bar{R}+G R \bar{G} \bar{R}) \\
& +\frac{1}{2}(B R \bar{B} \bar{R}+R B \bar{B} \bar{R}+B R \bar{R} \bar{B}+R B \bar{R} \bar{B}) \\
& \left.+\frac{1}{2}(G B \bar{G} \bar{B}+B G \bar{G} \bar{B}+G B \bar{B} \bar{G}+B G \bar{B} \bar{G})\right] \\
& \psi_{[11] \overline{3}[211]_{3}^{c}}^{q_{1} c_{2} \bar{q}_{3} \bar{c}_{4}}=\frac{1}{\sqrt{3}}\left[\frac{1}{2}\left(R_{1} G_{2}-G_{1} R_{2}\right)\left(\bar{R}_{3} \bar{G}_{4}-\bar{G}_{3} \bar{R}_{4}\right)\right. \\
& +\frac{1}{2}\left(B_{1} R_{2}-R_{1} B_{2}\right)\left(\bar{B}_{3} \bar{R}_{4}-\bar{R}_{3} \bar{B}_{4}\right) \\
& \left.+\frac{1}{2}\left(G_{1} B_{2}-B_{1} G_{2}\right)\left(\bar{G}_{3} \bar{B}_{4}-\bar{B}_{3} \bar{G}_{4}\right)\right] \\
& =\frac{1}{\sqrt{3}}\left[\frac{1}{2}(R G \bar{R} \bar{G}-G R \bar{R} \bar{G}-R G \bar{G} \bar{R}+G R \bar{G} \bar{R})\right. \\
& +\frac{1}{2}(B R \bar{B} \bar{R}-R B \bar{B} \bar{R}-B R \bar{R} \bar{B}+R B \bar{R} \bar{B}) \\
& \left.+\frac{1}{2}(G B \bar{G} \bar{B}-B G \bar{G} \bar{B}-G B \bar{B} \bar{G}+B G \bar{B} \bar{G})\right] \text {. }
\end{aligned}
$$

The explicit spin wave functions $\psi_{(S(q c) \otimes S(\bar{q} \bar{c}))}^{S(q c \bar{c})}$ of $q c \bar{q} \bar{c}$ tetraquark states are listed as follows:

$$
\begin{aligned}
& \psi_{(1 \otimes 1)}^{S=2}=\uparrow \uparrow \uparrow \bar{\uparrow}, \\
& \psi_{(1 \otimes 1)}^{S=1}=\frac{1}{2}(\uparrow \uparrow \uparrow \bar{\downarrow}+\uparrow \uparrow \bar{\downarrow} \bar{\uparrow}-\uparrow \downarrow \bar{\uparrow} \bar{\uparrow}-\downarrow \uparrow \uparrow \uparrow \bar{\uparrow}), \\
& \psi_{(1 \otimes 0)}^{S=1}=\frac{1}{\sqrt{2}}(\uparrow \uparrow \uparrow \bar{\downarrow}-\uparrow \uparrow \bar{\downarrow} \bar{\uparrow}), \\
& \psi_{(1 \otimes 1)}^{S=0}=\frac{1}{\sqrt{3}}\left[\uparrow \uparrow \bar{\downarrow} \bar{\downarrow}-\frac{1}{2}(\uparrow \downarrow \uparrow \bar{\downarrow}+\uparrow \downarrow \bar{\downarrow} \bar{\uparrow}+\downarrow \uparrow \bar{\uparrow} \bar{\downarrow}+\downarrow \uparrow \bar{\downarrow} \bar{T})\right. \\
& +\downarrow \downarrow \uparrow \bar{\uparrow}], \\
& \psi_{(0 \otimes 0)}^{S=0}=\frac{1}{2}(\uparrow \downarrow \uparrow \bar{\downarrow}-\uparrow \downarrow \bar{\downarrow} \bar{\uparrow}-\downarrow \uparrow \uparrow \bar{\downarrow}+\downarrow \uparrow \bar{\downarrow} \bar{\uparrow}) .
\end{aligned}
$$

The explicit spin wave function $\psi_{(S(c c) \otimes S(\bar{c} \bar{c}))}^{S(c c \bar{c})}$ of $c c \bar{c} \bar{c}$ $[2]\left(c_{1} c_{2}\right) \otimes[22]\left(\bar{c}_{3} \bar{c}_{4}\right)$ configuration is listed as follows: 
TABLE XII. The complete bases of tetraquark with quantum number, $N=2 n$ and $L=M=0$.

\begin{tabular}{|c|c|}
\hline$\psi_{400}$ & $\begin{array}{l}\psi_{2,0,0}\left(\vec{\sigma}_{1}\right) \psi_{0,0,0}\left(\vec{\sigma}_{2}\right) \psi_{0,0,0}(\vec{\lambda}), \psi_{0,0,0}\left(\vec{\sigma}_{1}\right) \psi_{2,0,0}\left(\vec{\sigma}_{2}\right) \psi_{0,0,0}(\vec{\lambda}), \psi_{0,0,0}\left(\vec{\sigma}_{1}\right) \psi_{0,0,0}\left(\vec{\sigma}_{2}\right) \psi_{2,0,0}(\vec{\lambda}), \psi_{1,0,0}\left(\vec{\sigma}_{1}\right) \psi_{1,0,0}\left(\vec{\sigma}_{2}\right) \psi_{0,0,0}(\vec{\lambda}) \\
\psi_{1,0,0}\left(\vec{\sigma}_{1}\right) \psi_{0,0,0}\left(\vec{\sigma}_{2}\right) \psi_{1,0,0}(\vec{\lambda}), \psi_{0,0,0}\left(\vec{\sigma}_{1}\right) \psi_{1,0,0}\left(\vec{\sigma}_{2}\right) \psi_{1,0,0}(\vec{\lambda})\end{array}$ \\
\hline$\psi_{600}$ & $\begin{array}{l}\psi_{3,0,0}\left(\vec{\sigma}_{1}\right) \psi_{0,0,0}\left(\vec{\sigma}_{2}\right) \psi_{0,0,0}(\vec{\lambda}), \psi_{2,0,0}\left(\vec{\sigma}_{1}\right) \psi_{1,0,0}\left(\vec{\sigma}_{2}\right) \psi_{0,0,0}(\vec{\lambda}), \psi_{2,0,0}\left(\vec{\sigma}_{1}\right) \psi_{0,0,0}\left(\vec{\sigma}_{2}\right) \psi_{1,0,0}(\vec{\lambda}), \psi_{1,0,0}\left(\vec{\sigma}_{1}\right) \psi_{2,0,0}\left(\vec{\sigma}_{2}\right) \psi_{0,0,0}(\vec{\lambda}), \\
\psi_{1,0,0}\left(\vec{\sigma}_{1}\right) \psi_{0,0,0}\left(\vec{\sigma}_{2}\right) \psi_{2,0,0}(\vec{\lambda}), \psi_{0,0,0}\left(\vec{\sigma}_{1}\right) \psi_{3,0,0}\left(\vec{\sigma}_{2}\right) \psi_{0,0,0}(\vec{\lambda}), \psi_{0,0,0}\left(\vec{\sigma}_{1}\right) \psi_{2,0,0}\left(\vec{\sigma}_{2}\right) \psi_{1,0,0}(\vec{\lambda}), \psi_{0,0,0}\left(\vec{\sigma}_{1}\right) \psi_{1,0,0}\left(\vec{\sigma}_{2}\right) \psi_{2,0,0}(\vec{\lambda}), \\
\psi_{0,0,0}\left(\vec{\sigma}_{1}\right) \psi_{0,0,0}\left(\vec{\sigma}_{2}\right) \psi_{3,0,0}(\vec{\lambda}), \psi_{1,0,0}\left(\vec{\sigma}_{1}\right) \psi_{1,0,0}\left(\vec{\sigma}_{2}\right) \psi_{1,0,0}(\vec{\lambda})\end{array}$ \\
\hline$\psi_{800}$ & $\begin{array}{l}\psi_{4,0,0}\left(\vec{\sigma}_{1}\right) \psi_{0,0,0}\left(\vec{\sigma}_{2}\right) \psi_{0,0,0}(\vec{\lambda}), \psi_{3,0,0}\left(\vec{\sigma}_{1}\right) \psi_{1,0,0}\left(\vec{\sigma}_{2}\right) \psi_{0,0,0}(\vec{\lambda}), \psi_{3,0,0}\left(\vec{\sigma}_{1}\right) \psi_{0,0,0}\left(\vec{\sigma}_{2}\right) \psi_{1,0,0}(\vec{\lambda}), \psi_{2,0,0}\left(\vec{\sigma}_{1}\right) \psi_{2,0,0}\left(\vec{\sigma}_{2}\right) \psi_{0,0,0}(\vec{\lambda}), \\
\psi_{2,0,0}\left(\vec{\sigma}_{1}\right) \psi_{1,0,0}\left(\vec{\sigma}_{2}\right) \psi_{1,0,0}(\vec{\lambda}), \psi_{2,0,0}\left(\vec{\sigma}_{1}\right) \psi_{0,0,0}\left(\vec{\sigma}_{2}\right) \psi_{1,0,0}(\vec{\lambda}), \psi_{1,0,0}\left(\vec{\sigma}_{1}\right) \psi_{3,0,0}\left(\vec{\sigma}_{2}\right) \psi_{0,0,0}(\vec{\lambda}), \psi_{1,0,0}\left(\vec{\sigma}_{1}\right) \psi_{2,0,0}\left(\vec{\sigma}_{2}\right) \psi_{1,0,0}(\vec{\lambda}) \\
\psi_{1,0,0}\left(\vec{\sigma}_{1}\right) \psi_{1,0,0}\left(\vec{\sigma}_{2}\right) \psi_{2,0,0}(\vec{\lambda}), \psi_{1,0,0}\left(\vec{\sigma}_{1}\right) \psi_{0,0,0}\left(\vec{\sigma}_{2}\right) \psi_{3,0,0}(\vec{\lambda}), \psi_{0,0,0}\left(\vec{\sigma}_{1}\right) \psi_{4,0,0}\left(\vec{\sigma}_{2}\right) \psi_{0,0,0}(\vec{\lambda}), \psi_{0,0,0}\left(\vec{\sigma}_{1}\right) \psi_{3,0,0}\left(\vec{\sigma}_{2}\right) \psi_{1,0,0}(\vec{\lambda}), \\
\psi_{0,0,0}\left(\vec{\sigma}_{1}\right) \psi_{2,0,0}\left(\vec{\sigma}_{2}\right) \psi_{2,0,0}(\vec{\lambda}), \psi_{0,0,0}\left(\vec{\sigma}_{1}\right) \psi_{1,0,0}\left(\vec{\sigma}_{2}\right) \psi_{3,0,0}(\vec{\lambda}), \psi_{0,0,0}\left(\vec{\sigma}_{1}\right) \psi_{0,0,0}\left(\vec{\sigma}_{2}\right) \psi_{4,0,0}(\vec{\lambda})\end{array}$ \\
\hline$\psi_{1000}$ & $\begin{array}{l}\psi_{5,0,0}\left(\vec{\sigma}_{1}\right) \psi_{0,0,0}\left(\vec{\sigma}_{2}\right) \psi_{0,0,0}(\vec{\lambda}), \psi_{4,0,0}\left(\vec{\sigma}_{1}\right) \psi_{1,0,0}\left(\vec{\sigma}_{2}\right) \psi_{0,0,0}(\vec{\lambda}), \psi_{4,0,0}\left(\vec{\sigma}_{1}\right) \psi_{0,0,0}\left(\vec{\sigma}_{2}\right) \psi_{1,0,0}(\vec{\lambda}), \psi_{3,0,0}\left(\vec{\sigma}_{1}\right) \psi_{2,0,0}\left(\vec{\sigma}_{2}\right) \psi_{0,0,0}(\vec{\lambda}), \\
\psi_{3,0,0}\left(\vec{\sigma}_{1}\right) \psi_{1,0,0}\left(\vec{\sigma}_{2}\right) \psi_{1,0,0}(\vec{\lambda}), \psi_{3,0,0}\left(\vec{\sigma}_{1}\right) \psi_{0,0,0}\left(\vec{\sigma}_{2}\right) \psi_{2,0,0}(\vec{\lambda}), \psi_{2,0,0}\left(\vec{\sigma}_{1}\right) \psi_{3,0,0}\left(\vec{\sigma}_{2}\right) \psi_{0,0,0}(\vec{\lambda}), \psi_{2,0,0}\left(\vec{\sigma}_{1}\right) \psi_{2,0,0}\left(\vec{\sigma}_{2}\right) \psi_{1,0,0}(\vec{\lambda}), \\
\psi_{2,0,0}\left(\vec{\sigma}_{1}\right) \psi_{1,0,0}\left(\vec{\sigma}_{2}\right) \psi_{1,0,0}(\vec{\lambda}), \psi_{2,0,0}\left(\vec{\sigma}_{1}\right) \psi_{0,0,0}\left(\vec{\sigma}_{2}\right) \psi_{3,0,0}(\vec{\lambda}), \psi_{1,0,0}\left(\vec{\sigma}_{1}\right) \psi_{4,0,0}\left(\vec{\sigma}_{2}\right) \psi_{0,0,0}(\vec{\lambda}), \psi_{1,0,0}\left(\vec{\sigma}_{1}\right) \psi_{3,0,0}\left(\vec{\sigma}_{2}\right) \psi_{1,0,0}(\vec{\lambda}), \\
\psi_{1,0,0}\left(\vec{\sigma}_{1}\right) \psi_{2,0,0}\left(\vec{\sigma}_{2}\right) \psi_{2,0,0}(\vec{\lambda}), \psi_{1,0,0}\left(\vec{\sigma}_{1}\right) \psi_{1,0,0}\left(\vec{\sigma}_{2}\right) \psi_{3,0,0}(\vec{\lambda}), \psi_{1,0,0}\left(\vec{\sigma}_{1}\right) \psi_{0,0,0}\left(\vec{\sigma}_{2}\right) \psi_{4,0,0}(\vec{\lambda}), \psi_{0,0,0}\left(\vec{\sigma}_{1}\right) \psi_{5,0,0}\left(\vec{\sigma}_{2}\right) \psi_{0,0,0}(\vec{\lambda}), \\
\psi_{0,0,0}\left(\vec{\sigma}_{1}\right) \psi_{4,0,0}\left(\vec{\sigma}_{2}\right) \psi_{1,0,0}(\vec{\lambda}), \psi_{0,0,0}\left(\vec{\sigma}_{1}\right) \psi_{3,0,0}\left(\vec{\sigma}_{2}\right) \psi_{2,0,0}(\vec{\lambda}), \psi_{0,0,0}\left(\vec{\sigma}_{1}\right) \psi_{2,0,0}\left(\vec{\sigma}_{2}\right) \psi_{3,0,0}(\vec{\lambda}), \psi_{0,0,0}\left(\vec{\sigma}_{1}\right) \psi_{1,0,0}\left(\vec{\sigma}_{2}\right) \psi_{4,0,0}(\vec{\lambda}), \\
\psi_{0,0,0}\left(\vec{\sigma}_{1}\right) \psi_{0,0,0}\left(\vec{\sigma}_{2}\right) \psi_{5,0,0}(\vec{\lambda})\end{array}$ \\
\hline
\end{tabular}

$\psi_{(0 \otimes 0)}^{S=0}=\frac{1}{2}(\uparrow \downarrow \uparrow \bar{\downarrow}-\uparrow \downarrow \bar{\downarrow} \uparrow-\downarrow \uparrow \uparrow \bar{\downarrow}+\downarrow \uparrow \bar{\downarrow} \bar{\uparrow})$.

The explicit spin wave functions $\psi_{(S(c c) \otimes S(\bar{c} \bar{c}))}^{S(c \bar{c} \bar{c})}$ of $c c \bar{c} \bar{c}$ $[11]\left(c_{1} c_{2}\right) \otimes[211]\left(\bar{c}_{3} \bar{c}_{4}\right)$ configuration are listed as follows:

$$
\begin{aligned}
& \psi_{(1 \otimes 1)}^{S=2}=\uparrow \uparrow \uparrow \uparrow, \\
& \psi_{(1 \otimes 1)}^{S=1}=\frac{1}{2}(\uparrow \uparrow \uparrow \bar{\downarrow}+\uparrow \uparrow \uparrow \bar{\downarrow} \uparrow-\uparrow \downarrow \uparrow \bar{\uparrow}-\downarrow \uparrow \uparrow \bar{\uparrow} \text { ), } \\
& \psi_{(1 \otimes 1)}^{S=0}=\frac{1}{\sqrt{3}}\left[\uparrow \uparrow \bar{\downarrow} \bar{\downarrow}-\frac{1}{2}(\uparrow \downarrow \uparrow \bar{\downarrow}+\uparrow \downarrow \bar{\downarrow} \uparrow+\downarrow \uparrow \uparrow \bar{\downarrow}+\downarrow \uparrow \bar{\downarrow} \uparrow)\right. \\
& +\downarrow \downarrow \uparrow \bar{\uparrow}] \text {. }
\end{aligned}
$$

The total spatial wave function of tetraquark, coupling among the $\sigma_{1}, \sigma_{2}$ and $\lambda$ harmonic oscillator wave functions may take the general form

$$
\begin{aligned}
\psi_{N L M}= & \sum_{\left\{n_{i}, l_{i}\right\}} A\left(n_{\sigma_{1}}, n_{\sigma_{2}}, n_{\lambda}, l_{\sigma_{1}}, l_{\sigma_{2}}, l_{\lambda}\right) \\
& \times \psi_{n_{\sigma_{1}} l_{\sigma_{1}}}\left(\vec{\sigma}_{1}\right) \otimes \psi_{n_{\sigma_{2}} l_{\sigma_{2}}}\left(\vec{\sigma}_{2}\right) \otimes \psi_{n_{\lambda} l_{\lambda}}(\vec{\lambda}) \\
= & \sum_{\left\{n_{i}, l_{i}, m_{i}\right\}} C_{n_{\sigma_{1}}, l_{\sigma_{1}}, m_{\sigma_{1}}, n_{\sigma_{2}}, l_{\sigma_{2}}, m_{\sigma_{2}}, n_{\lambda}, l_{\lambda}, m_{\lambda}} \\
& \times \psi_{n_{\sigma_{1}} l_{\sigma_{1}} m_{\sigma_{1}}}\left(\vec{\sigma}_{1}\right) \psi_{n_{\sigma_{2}} l_{\sigma_{2}} m_{\sigma_{2}}}(\vec{\eta}) \psi_{n_{\lambda} l_{\lambda} m_{\lambda}}(\vec{\lambda}),
\end{aligned}
$$

where $\psi_{n_{i} l_{i} m_{i}}$ are just harmonic oscillator wave functions and the sum $\left\{n_{i}, l_{i}\right\}$ is over $n_{\sigma_{1}}, n_{\sigma_{2}}, n_{\lambda}, l_{\sigma_{1}}$, $l_{\sigma_{2}}, l_{\lambda} . N, L$, and $M$ are respectively the total principle quantum number, total angular momentum, and magnetic quantum number of the tetraquark. One has $N=\left(2 n_{\sigma_{1}}+l_{\sigma_{1}}\right)+\left(2 n_{\sigma_{2}}+l_{\sigma_{2}}\right)+\left(2 n_{\lambda}+l_{\lambda}\right)$.

The complete bases of the tetraquarks are listed in Table XII up to $N=10$, where $l_{\sigma_{1}}, l_{\sigma_{2}}$, and $l_{\lambda}$ are limited to 0 only.
[1] M. Gell-Mann, Phys. Lett. 8, 214 (1964).

[2] G. Zweig, An SU(3) model for strong interaction symmetry and its breaking. Version 2, in Developments in the QUARK Theory of Hardons (CERN, Geneva, 1964), Vol. 1, pp. 22-101.

[3] N. Brambilla, S. Eidelman, C. Hanhart, A. Nefediev, C.-P. Shen, C. E. Thomas, A. Vairo, and C.-Z. Yuan, Phys. Rep. 873, 1 (2020).
[4] P. Zyla et al. (Particle Data Group Collaboration), Prog. Theor. Exp. Phys. 2020, 083C01 (2020).

[5] K. Chilikin et al. (Belle Collaboration), Phys. Rev. D 95, 112003 (2017).

[6] K. Abe et al. (Belle Collaboration), Phys. Rev. Lett. 94, 182002 (2005).

[7] P. Pakhlov et al. (Belle Collaboration), Phys. Rev. Lett. 100, 202001 (2008). 
[8] C. Shen et al. (Belle Collaboration), Phys. Rev. Lett. 104, 112004 (2010).

[9] R. Aaij et al. (LHCb Collaboration), Phys. Rev. Lett. 118, 022003 (2017).

[10] M. Ablikim et al. (BESIII Collaboration), Phys. Rev. Lett. 114, 092003 (2015).

[11] B. Aubert et al. (BABAR Collaboration), Phys. Rev. Lett. 95, 142001 (2005).

[12] M. Ablikim et al. (BESIII Collaboration), Phys. Rev. D 96, 032004 (2017).

[13] M. Ablikim et al. (BESIII Collaboration), Phys. Rev. Lett. 118, 092002 (2017).

[14] X. Wang et al. (Belle Collaboration), Phys. Rev. D 91, 112007 (2015).

[15] M. Ablikim et al. (BESIII Collaboration), Phys. Rev. Lett. 112, 022001 (2014).

[16] M. Ablikim et al. (BESIII Collaboration), Phys. Rev. Lett. 111, 242001 (2013).

[17] R. Mizuk et al. (Belle Collaboration), Phys. Rev. D 78, 072004 (2008).

[18] R. Aaij et al. (LHCb Collaboration), Eur. Phys. J. C 78, 1019 (2018).

[19] K. Chilikin et al. (Belle Collaboration), Phys. Rev. D 90, 112009 (2014).

[20] L. Maiani, F. Piccinini, A. D. Polosa, and V. Riquer, Phys. Rev. D 71, 014028 (2005).

[21] L. Maiani, V. Riquer, R. Faccini, F. Piccinini, A. Pilloni, and A. D. Polosa, Phys. Rev. D 87, 111102(R) (2013).

[22] L. Maiani, F. Piccinini, A. D. Polosa, and V. Riquer, Phys. Rev. D 89, 114010 (2014).

[23] S. Patel, M. Shah, and P. C. Vinodkumar, Eur. Phys. J. A 50, 131 (2014).

[24] L. Zhao, W.-Z. Deng, and S.-L. Zhu, Phys. Rev. D 90, 094031 (2014).

[25] K. Xu, A. Kaewsnod, X. Y. Liu, S. Srisuphaphon, A. Limphirat, and Y. Yan, Phys. Rev. C 100, 065207 (2019).

[26] Y. Ikeda and H. Iida, Prog. Theor. Phys. 128, 941 (2012).

[27] T. Kawanai and S. Sasaki, Phys. Rev. Lett. 107, 091601 (2011).

[28] G. S. Bali, Phys. Rep. 343, 1 (2001).

[29] R. M. Albuquerque, J. M. Dias, K. Khemchandani, A. Martínez Torres, F. S. Navarra, M. Nielsen, and C. M. Zanetti, J. Phys. G 46, 093002 (2019).

[30] C. Deng, J. Ping, H. Huang, and F. Wang, Phys. Rev. D 92, 034027 (2015).

[31] D. Ebert, R. Faustov, and V. Galkin, Eur. Phys. J. C 58, 399 (2008).

[32] C. Deng, J. Ping, H. Huang, and F. Wang, Phys. Rev. D 98, 014026 (2018).

[33] Z. Guo, T. Liu, and B.-Q. Ma, Phys. Rev. D 93, 076010 (2016).

[34] F. Goerke, T. Gutsche, M. A. Ivanov, J. G. Korner, V. E. Lyubovitskij, and P. Santorelli, Phys. Rev. D 94, 094017 (2016).
[35] Z.-G. Wang, Commun. Theor. Phys. 63, 325 (2015).

[36] S. S. Agaev, K. Azizi, and H. Sundu, Phys. Rev. D 96, 034026 (2017).

[37] C. Deng, J. Ping, H. Huang, and F. Wang, Phys. Rev. D 90, 054009 (2014).

[38] M. Ablikim et al. (BESIII Collaboration), Phys. Rev. Lett. 112, 132001 (2014).

[39] M. Ablikim et al. (BESIII Collaboration), Phys. Rev. Lett. 113, 212002 (2014).

[40] M. Ablikim et al. (BESIII Collaboration), Phys. Rev. Lett. 115, 182002 (2015).

[41] A. Bondar, Complementarity of the beauty and charm hadron physics, in Proceedings of the 9th International Workshop on Charm Physics, 2018, Novosibirsk, Russia (2018), https://indico.inp.nsk.su/event/10/contributions/ 292/attachments/199/231/A.Bondar_Charm18.pdf.

[42] J. Wu, X. Liu, Y.-R. Liu, and S.-L. Zhu, Phys. Rev. D 99, 014037 (2019).

[43] L. Zhao, L. Ma, and S.-L. Zhu, Phys. Rev. D 89, 094026 (2014).

[44] J. He, Phys. Rev. D 90, 076008 (2014).

[45] S. Prelovsek and L. Leskovec, Phys. Lett. B 727, 172 (2013).

[46] Y. Chen, M. Gong, Y. H. Lei, N. Li, J. Liang, C. Liu, H. Liu, J. L. Liu, L. Liu, Y. F. Liu, Y. B. Liu, Z. Liu, J. P. Ma, Z. L. Wang, Y. B. Yang, and J.B. Zhang, Phys. Rev. D 89, 094506 (2014).

[47] Z.-G. Wang and T. Huang, Eur. Phys. J. C 74, 2891 (2014).

[48] F. Aceti, M. Bayar, E. Oset, A. M. Torres, K. P. Khemchandani, J. M. Dias, F. S. Navarra, and M. Nielsen, Phys. Rev. D 90, 016003 (2014).

[49] H.-W. Ke and X.-Q. Li, Eur. Phys. J. C 76, 334 (2016).

[50] R. Aaij et al. (LHCb Collaboration), Sci. Bull. 65, 1983 (2020).

[51] G. Yang, J. Ping, L. He, and Q. Wang, arXiv:2006.13756.

[52] G.-J. Wang, L. Meng, and S.-L. Zhu, Phys. Rev. D 100, 096013 (2019).

[53] M.-S. Liu, Q.-F. Lü, X.-H. Zhong, and Q. Zhao, Phys. Rev. D 100, 016006 (2019).

[54] R. J. Lloyd and J. P. Vary, Phys. Rev. D 70, 014009 (2004).

[55] J. P. Ader, J. M. Richard, and P. Taxil, Phys. Rev. D 25, 2370 (1982).

[56] H.-X. Chen, W. Chen, X. Liu, and S.-L. Zhu, Sci. Bull. 65, 1994 (2020).

[57] N. Barnea, J. Vijande, and A. Valcarce, Phys. Rev. D 73, 054004 (2006).

[58] A. V. Berezhnoy, A. V. Luchinsky, and A. A. Novoselov, Phys. Rev. D 86, 034004 (2012).

[59] Z.-G. Wang, Eur. Phys. J. C 77, 432 (2017).

[60] Z.-G. Wang and Z.-Y. Di, Acta Phys. Pol. B 50, 1335 (2019).

[61] V. Debastiani and F. Navarra, Chin. Phys. C 43, 013105 (2019). 